\title{
Design and Implementation of a Fully Automatic Assembling System for Automobile Fuel Tank Overturn Valve
}

\author{
Zhiming Wang ${ }^{1}$,Changfu Pang ${ }^{1, a}$,Zhenglong Cai ${ }^{1}$ and Lizhen Cui ${ }^{1}$ \\ School of Mechatronic Engineering and Automation, Shanghai University, Shanghai 200072, China
}

\begin{abstract}
At present, at least one or two overturn valves are required in each automobile production process. However, the production and assembly of overturn valve is still in the stage of manual assembly, which leads to low production efficiency. This paper mainly designs a fully automatic assembly system based on the structure of the overturn valve. The automatic assembly system includes loading, assembly, transportation of blanks and other work stations, and automatic assembly based on the PLC control system. At the same time, the human interaction page touch screen is used for data monitoring and parameter setting. The experimental results show that the assembly system can complete the automatic assembly of the overturning valve of the fuel tank stably and reliably
\end{abstract}

\section{Introduction}

Tank overturn valves are used more and more widely in the automotive industry. Their role is to prevent the fuel in the fuel tank from flowing into the exhaust pipe when the vehicle is tilting or overturning. This prevents the fuel from exploding. Therefore, the overturn valve is a key guarantee for safe driving of the vehicle[1]. Assembly quality often plays an important role in the performance of rollover valve. However, assembly is much more complicated than machining, assembly methods often vary from product, and the assembly method chosen is incorrect, which can lead to a decrease in assembly success rate and affect the assembly quality of the product[2].

This paper mainly studies the assembly of overturn valve cores and realizes automatic assembly of the valve cores. According to the requirements of the valve core assembly unit and the characteristics of the assembly objects, the overall structure of the system is designed in conjunction with relevant advanced technologies. The general structure of assembly process and machinery is taken as the guide to determine the control system. In the aspect of software design, the upper computer program is designed by using WEINVIEW touch screen, and the automatic assembly system is debugged through communication with Siemens PLC.

\section{Structural Design of Overturning Valve Assembly System}

Automatic assembly is actually a process of connecting and combining a group of scattered parts through reasonable process flow and various necessary methods according to the determined accuracy standards and technical conditions[3]. A product has a variety of optional assembly sequences. Different assembly sequences will have different assembly methods and have different mechanical structures. After experiments combined with theoretical analysis, it is determined that the order of assembly parts of the overturn valve core is float, film and bridge.

\subsection{General layout of spool assembly unit}

The automatic assembly system of overturn valve core consists of four parts: work station, protective cover, vibration feeding table and control system. According to the different type of overturn valve, different feeding stations and jigs are designed to meet different product requirements.

In the process of actual assembly and production, there are six feeding vibratory trays. According to the size of the overturn valve core, only three feeding trays are used in a single use. Before changing the products, all the tools on the turntable will be replaced with the matching molds for the products to be produced. It should be noted that in order to make the structure compact and at the same time reduce the cost, the product handling station is designed as a public station and can be replaced without replacement.

The overall structure of the automatic assembly machine is shown in the figure below: 


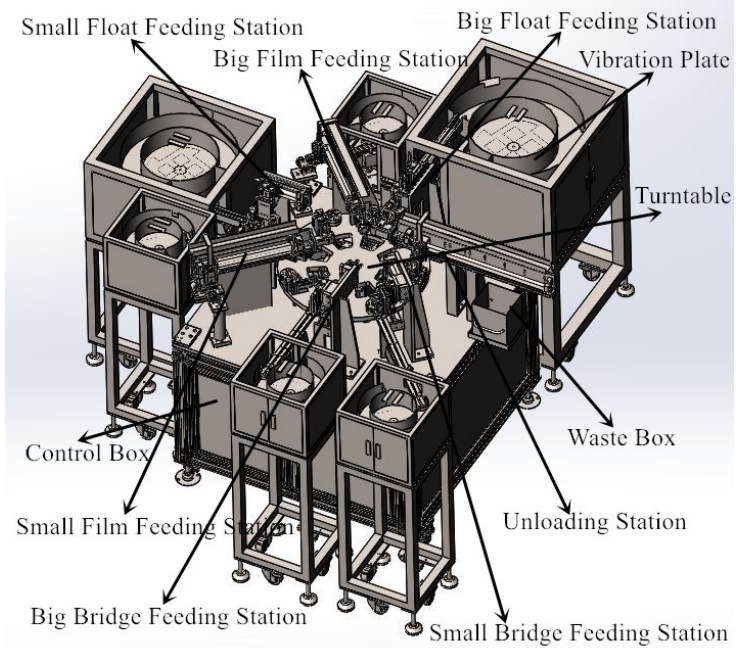

Figure 1.Layout diagram of automatic assembly machine

\subsection{Design of automatic feeding system for parts}

The parts supply system consists of five steps: storage, alignment, feeding, separation, and material management. According to the actual situation, the automatic feeding vibration plate completes the storage of parts, and its internal spiral structure sorts and arranges the parts, and has a reliable discharging state to realize the automatic supply of parts. The linear feeding vibration completes the long-distance feeding of the parts, allowing the parts to enter the assembly area. The separation device separates the parts to be assembled by gas grasp, so that the parts can be sorted or grabbed. The sensor on the tool mold detects the positive and negative of the material, and the rotating material management device completes the change of the positive and negative status of the component, so that the component has the state of capturing to the assembly station.

\subsection{Design of conveying turntable structure}

Because the spool assembly process is less, use the mode of synchronous transportation as far as possible. By comparing the basic types of several parts and components transportation, it is determined to select the rotary indexing mode of transportation to complete the assembly of a component. Its application in the automatic assembly machine is mostly 8 to 10 workstations, and under the control of PLC and touch screen software to achieve periodic movements, accurate indexing, smooth transmission, high efficiency, can meet the actual production needs.

\section{Scheme Design of Control system}

The control system of the assembly line, like the human brain, controls the operation of the entire production line and plays a crucial role in the normal operation, reliability, and troubleshooting of the assembly line[4].
The bottom control part of the automatic assembly unit of the valve core is completed by PLC software, and

the monitoring and parameter settings are completed by the touch screen.

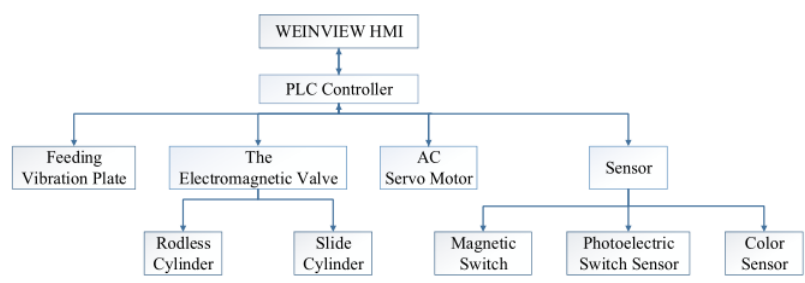

Figure 2.Equipment control mode diagram

\subsection{Design of PLC Control Software}

As an organic whole, the rotary table automatic assembly machine is composed of several parts, such as parts automatic supply system, handling system, assembly parts, assembly components, assembly products and sorting products. The control system is mainly coordinated by PLC controller, servo motor, cylinder and other actuators to realize automatic assembly[5].

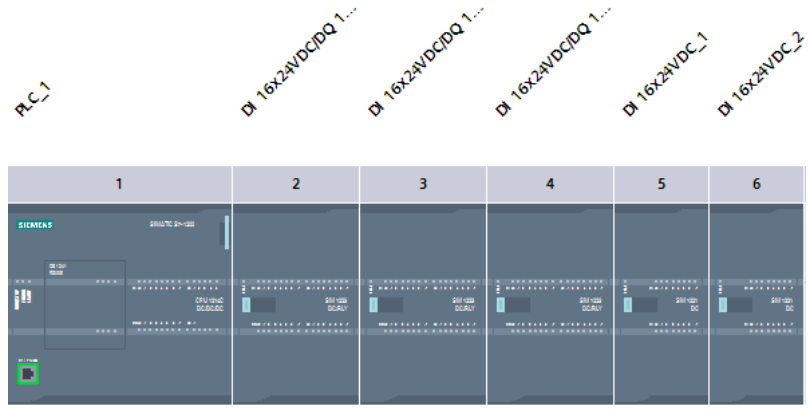

Figure 3.Equipment configuration view

According to the actual assembly production requirements, the control system of the overturn valve assembly unit is designed with two modes of operation: manual mode and automatic mode. The manual mode includes single-station testing and single-step testing. In addition, the system is designed with an initialization module, an alarm module, and a fault processing module.

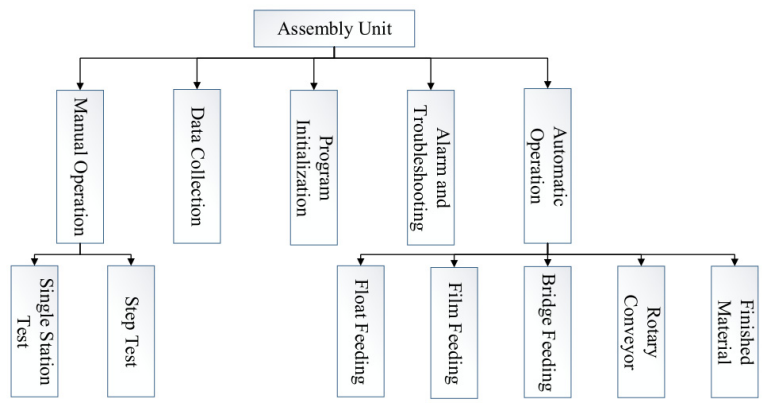

Figure 4. Assembly system control module diagram

\subsubsection{Program initialization module}

This module performs the assignment operation to the intermediate relays used in the program every time the PLC is powered off and restarted. After the initialization 
of the program, the main actions are the turning of the cylinder, suction of the sucker and so on.

\subsubsection{Data collection}

The module mainly collects sensor signals and processes them. The main collection of the cylinder upper and lower limit signal, servo motor position signal and the state signal of conveying turntable. When the PLC executes each cycle scan, the collected signals are compared with the corresponding actuator states, so that corresponding actions are performed to achieve the switching of the states.

\subsubsection{Automatic operation module}

The module mainly includes four processes: feeding on the float, pressing on the film, feeding on the bridge, conveying on the turntable, and unloading the finished product. The module judges the operating status of the system based on the data collected by the data acquisition module. After processing feedback from the PLC central processing station, the actuators such as the servo motor, dial divider, pneumatic slide and cylinder will be operated in turn to complete the action of this station and realize automatic assembly.

\subsubsection{Manual operation module}

The module mainly includes single-station operation and single-step operation. The manual operation mode mainly performs the action of the corresponding station according to the command transmitted from the touch screen.

\subsubsection{Alarm and fault handling module}

This module is mainly used in the automatic assembly process of the valve core. According to the collected signal, when the status is abnormal, the system displays the corresponding alarm indication on the touch screen. According to the alarm indication on the touch screen, troubleshooting is performed to protect the normal operation of the system.

\subsection{Design of human-computer interaction page}

This assembly system mainly uses WEINVIEW touch screen as human-machine interface. The touch screen mainly monitors the assembly process in real time, and can perform manual operations and set some parameters. At the same time, the data in the PLC can be exported, the product can be counted, and the fault information can be found in time and eliminated.

\subsubsection{Human-machine interaction main interface}

The main interface is mainly for the monitoring of data and status. It can basically see all the information required for product assembly, including the number of qualified and unqualified products, product number, cycle time, alarms information, turntable status and realtime operation information of the equipment. It also includes manual operation and parameter setting button.

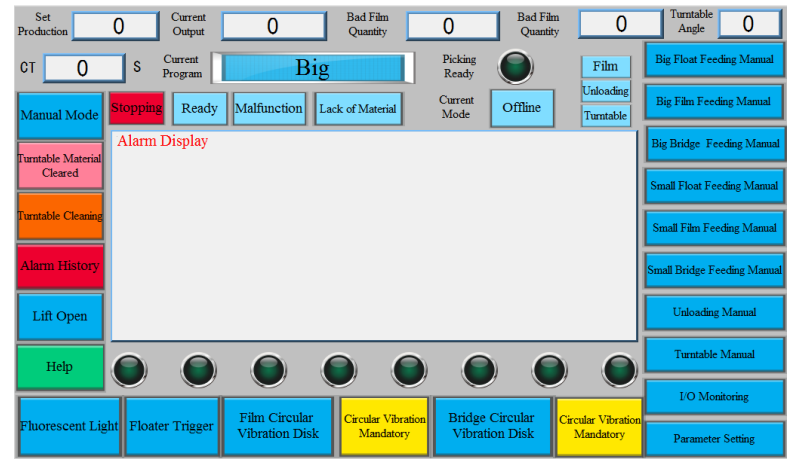

Figure 5.Touch screen main interface

\subsubsection{Parameter setting interface}

The device parameter setting interface mainly sets the parameters needed for the operation of the device, such as setting the position and speed of the assembly process, the type of the product and the lack of material alarm cycle and so on.

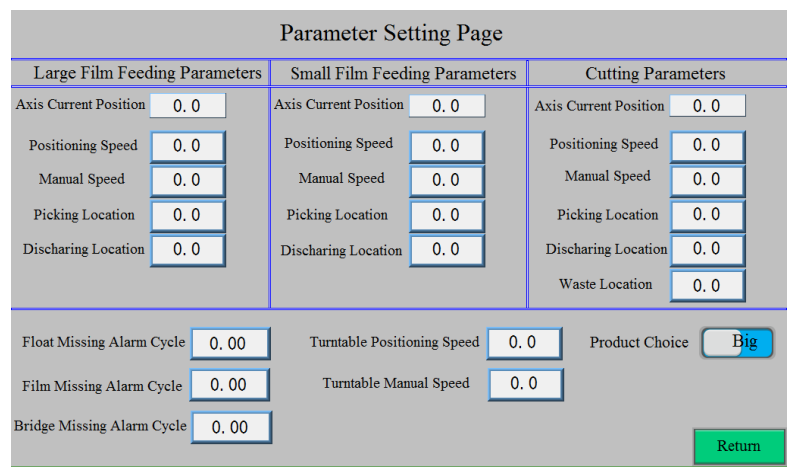

Figure 6.Touch screen parameter setting page

\subsubsection{Manual operation interface}

The manual operation interface is mainly used for debugging and setting of auxiliary equipment parameters. The interface mainly implements two functions. One part is the jog control of the loading cylinder, clamping cylinder and servo motor. The other part can be used for testing. There are two kinds of testing functions. One is a single-station test, which mainly completes the work flow of the station; the other is a single-step test, presses the next button in turn, this station follow the proper procedure for single-step operation. 


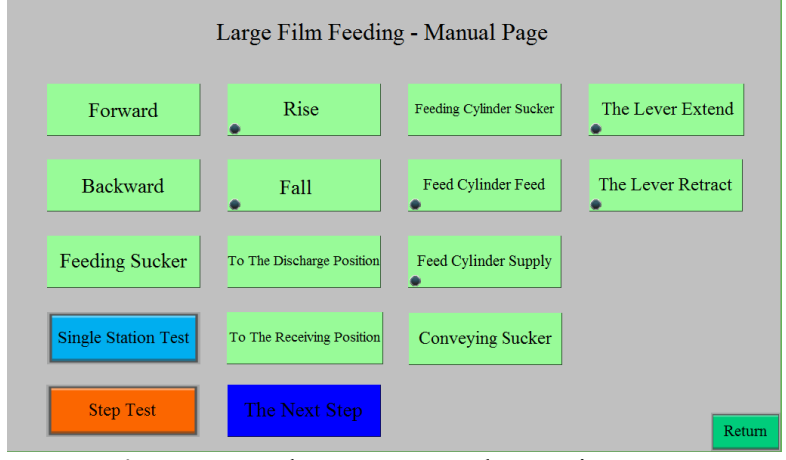

Figure 7.Touch screen manual operation page

\section{Communication}

\subsection{Ethernet communication between S7-1200 PLC and Touch Screen}

A PROFINET communication port is integrated on the S7-1200 PLC, supporting Ethernet and TCP/IP based communication standards. After assigning the IP address of the PLC and the touch screen, the touch screen is connected to the network cable with the PLC. After the PLC searches for accessible devices, confirm the connection to complete the communication.

The communication between the touch screen and the PLC is realized by the variables in the PLC. When generating variables, you must first check the "optimized block access" of the data block in the PLC, and then generate the source from the block to generate the tag. When creating a touch screen images, simply import the tags and select the elements in the user-defined global variables or global data blocks in the S7-1200 PLC.

\subsection{Communication between S7-1200 PLC and Servo Driver}

The S7-1200 expands the CB1241RS485 signal board through the CPU to realize the communication function of the S7-1200 master station. The serial port based on RS485 realizes free port communication between Siemens 1200 and three servo slave stations. In the "Port" configuration options, configure the port parameters, complete the hardware configuration, and write serial communication programs[6].

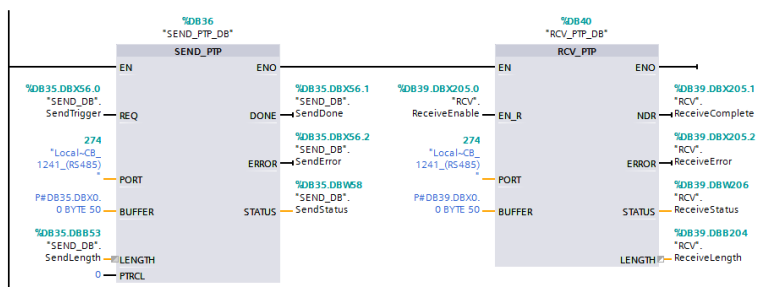

Figure 8.Serial communication program

\section{Experimental results and analysis}

According to actual production conditions, statistics of 1000 assembled finished products were obtained.
Through data processing and analysis, the normal operation cycle of a valve core was less than 4 seconds. Among them, the qualified rate reached $96 \%$, and the defects of defective products mainly focused on the film superposition, location bias, and missing bridges.

To solve the problem of defective products, the main improvement is from the following aspects. First, add multiple stages of vacuum suction nozzles on film feeding and conveying sites to avoid stacking. Second, adjust the position of the clamp and the stopper plate to ensure the accuracy of the placement. Finally, the speed of the servo motor is properly controlled and the ultrasonic welding machine is used to weld the finished products together to prevent the missing parts.

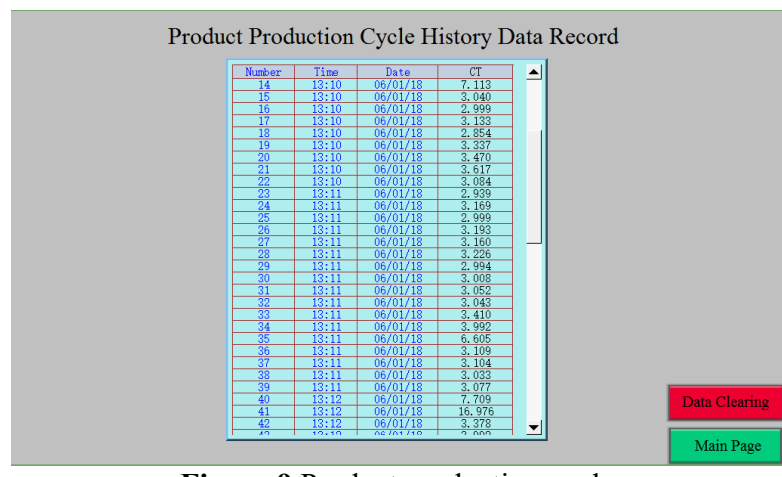

Figure 9.Product production cycle

Through the prototype test, the test results show that: the assembly system is a set of mechanical, pneumatic, PLC control as one of the precision assembly equipment.

\section{Conclusion}

This article mainly designs the automatic assembly system of automobile fuel tank overturn valve, mainly including the mechanical structure and the control system.

This system adopts the assembly method of turntable transport, adopts PLC as the controller of the system, the actuator adopts AC servo motor, cylinder and so on. In addition, it also adopts closed-loop control to obtain greater positioning accuracy, thereby ensuring assembly accuracy. At the time of design, multiple protection measures were taken on the system to prevent the system from damaging the device in case of accident. At the same time, the PLC control software was designed to realize the automatic operation of the control process. Based on the PLC control system, the touch screen monitoring software is designed and communicated with it, which can monitor the operation of the system in real time.

\section{References}

1. Wei Sun, Jiayuan Li. Fault Prediction of Tank Rollover Valve Based on Fuzzy Least Squares Support Vector Machine. Industrial Control and Computer, 2016, 29(12):78-79. 
2. Junfang Ni, Jianzhong Mo. Product Design Method for Assembly Automation. Journal of Suzhou Academy of Silk Sciences, 1999, 19(2):29-34.

3. Tinggang Sun. Research and Application of Key Technologies for Automatic Assembly Line (Machine) Design. Sichuan University, 2007.

4. Yongqiang Li. Research on automotive ABS cartridge automated assembly unit. College of Mechanical and Energy, Zhejiang University, Zhejiang University, 2007.

5. Yuelin Xu. Design of Control System of Rotary Disc Automatic Assembler. Journal of Electrical Engineering, 2011 (4):23-24.

6. Siemens Ltd. Easy to understand Siemens S7-1200 PLC. Beijing University of Aeronautics and Astronautics Press, 2009. 